gastroinestinal disease was observed only in one patient, cardiovascular and central nervous system involvement was respectively seen in 2 and 3 cases. Out of the 5 patients tested for ANCA, 2 resulted positive; prednisone treatment was started in 11 of the 14 patients at the mean dosage of $1 \mathrm{mg} / \mathrm{kg} / \mathrm{day}$, whereas at the 3 remaining patients was associated Cyclophosphamide at the dosage of $2 \mathrm{mg} / \mathrm{kg} /$ day. Only in one case IVIG pulse at the dosage of $1 \mathrm{gr} / \mathrm{kg} /$ for 5 days was started with good result.

The most recurrent side effects were urinary infections (3 cases), pulmonary infections (2 cases), tubercolosis ( 2 cases), diabetis ( 2 cases), Herpes Zoster (3 cases). Remissions were observed in all patients, relapses were seen though well controlled by the increase of the steroid therapy. 3 patients died: 1 for mesenteric vasculitis, 1 for respiratory insufficiency and the last one for reasons not correlated to the disease. As reported in letterature, also in our experience survival is influenced by the systemic involvement of the disease and by FFS (Five factors score: combination of renal, cardiac, neurologic, gastrointestinal involvement plus proteinuria). In our series survival at 120 months was $75 \%$, the worst prognostic index was seen in patients with FFS $>1$.

Objectives

Methods

Results

Conclusion

\section{FRI0255 POLYMYOSITIS AND DERMATOMYOSITIS: A REVIEW OF 47 CASES}

CC Ben Taarit, S Turki, E Abderrahim, H Ben Maiz. Nephrology and Internal Medicine, Charles Nicolle Hospital, Tunis, Tunisia

\subsection{6/annrheumdis-2001.576}

Background Polymyositis (PM) and Dermatomyositis (DM) are a multisystem disease characterised by acute and chronic nonsuppurative inflammation of striated muscle and skin.

Objectives Objectives of this study is to describe the clinical profile, course and treatment of 47 cases of PM and DM observed over a period of 26 years.

Methods Retrospective review of 47 cases with PM and DM.

Results There were 36 women and 11 men with a mean age of 35 years. The classification showed: 21 cases of PM and 16 cases of DM, one case of DM was associated with a malignancy, one case of DM in children and 8 cases of an overlapping syndrome. Muscle weakness and myalgia were constant, non destructive arthritis or arthralgia were observed in 32 cases, calcinosis in 2 patients (1 DM in children and 1 overlapping syndrome with scleroderma).

Results of biological tests, erythrocyte sedimentation rate and serum enzyme determination were abnormal in respectively 29 cases and 46 cases.

The electromyogram was generally of a myogenic type with spontaneous activity (39/41). Steroïd therapy is prescribed in all cases and additional immunosuppressive drug (methotrexate, cyclophosphamide) is performed in 15 cases.

Conclusion DM and PM are a multisystemic disease with good response to only steroïd therapy associated to immunosuppressive drugs.
FRI0256 LOCAL PULSED ULTRASOUND THERAPY VERSUS CYRIAX?S METHOD OF DEEP FRICTION-MANIPULATION IN THE TREATMENT OF LATERAL EPICONDYLITIS: PRELIMINARY REPORT

R Guzel, F Uysal, M Buyukkose, A Guney. Physical Medicine and Rehabilitation, Cukurova University Medical Faculty, Adana, Turkey

\subsection{6/annrheumdis-2001.577}

Background Although physical therapy measures are commonly used for the conservative management of lateral epicondylitis, limited randomised prospective studies exist comparing the effects of different approaches.

Objectives The aim of the study was to compare the efficacy of local pulsed ultrasound (US) therapy with deep friction-manipulation as advocated by Cyriax in the treatment of lateral epicondylitis.

Methods 36 lateral epicondylitis patients with a mean age of $50,42 \pm 8,71$ years and a mean disease duration of $3,14 \pm 2,38$ months were enrolled into the study. Patients were randomly allocated into two treatment groups: 1) Ultrasound application of $1.5 \mathrm{watt} / \mathrm{cm}^{2}$ for $5 \mathrm{~min} 2$ ) Cyriax technique of deep friction massage and Mills? manipulation. Use of analgesics or epicondylitis bracing were not allowed and the treatment intervention lasted for a maximum period of 2 weeks. The same physiotherapist carried out the treatments and the observer was blinded to the treatment modality. Activity modification and gentle range of motion, stretching exercises were recommended to both groups. Outcome measures were pain intensity measured by VAS, pain produced by pressure on lateral epicondyle and on resisted wrist extension, grip strength and Verhaar?s scoring system for treatment results of lateral epicondylitis.

Results At baseline, the groups were similar in terms of age, pain scores, and the duration of symptoms. After treatment, pain produced by pressure on the lateral epicondyle and on resisted wrist extension were found to be significantly decreased in both groups $(\mathrm{p}<0.01)$. Reduction in pain intensity measured on VAS was significant in the Cyriax group but not in the US group ( $p$ $<0.05$ and $\mathrm{p}>0.05$ respectively). The improvement in grip strength was significant in both groups, being $38,4 \%$ in the Cyriax group, and 19,3\% in the US group ( $<<0.001$ and $\mathrm{p}<$ $0.05)$. Percentage of patients that reached excellent or good results according to Verhaar?s criteria was $55 \%$ in the Cyriax and $33 \%$ in the US group. When the two treatment groups were compared, the decrease in pain, improvement in grip strength and Verhaar?s criteria seemed to be in favour of the Cyriax group but the differences were not statistically significant.

Conclusion Our preliminary results support the efficacy of two different conservative approaches in the treatment of lateral epicondylitis. Although Cyriax type physiotherapy consisting of deep friction massage and Mills? manipulation seemed to be a better treatment approach than US, the difference did not reach statistical significance. The study is still running for investigating the long term effects of both treatments with increasing number of cases at 12 months.

\section{REFERENCE}

1 Verhaar JAN, Walenkamp GHIM, Mameren $\mathrm{H}$, et al. Local corticosteroid injection versus Cyriax-type physiotherapy for tennis elbow. J Bone Joint Surg (Br) 1995; 77-B:128-32 